merized ground state for arbitrary values of $M$ and $\lambda$ (except $M=0$ ). The underlying reason is that the phonon fluctuations induce an effective electron-electron interaction of such a type that a CDW ground state is always produced. (That interaction is ineffective in the case $n=1$ for small coupling because of the Pauli exclusion principle.) This is accompanied by pairing of the spin-up and spin-down electrons. However, this conclusion is by no means inescapable. Preliminary numerical studies ${ }^{9}$ show that other forms of the electron-phonon coupling (which induce longer-range attraction) give a ground state with superconducting correlations. This has also been suggested from calculations based on perturbation theory. ${ }^{10}$ The MC method used in this paper offers the possibility of numerically studying complicated one-dimensional electron-phonon models (the inclusion of electron-electron interaction is straightforward) and thus investigating the rich variety of ground-state phases for such systems, without restriction to a perturbative regime.

One of us (J.H.) is indebted to D. Scalapino for raising his interest in this problem and for numerous stimulating discussions. We acknowledge helpful conversations with S. Kivelson, W. P. Su,
R. Sugar, N. Andrei, S. Shenker, K. Maki, M. Stone, and particularly J. R. Schrieffer. One of us (E.F.) thanks the Institute for Theoretical Physics for its kind hospitality during the summer of 1981 . This work was supported by the National Science Foundation under Grants No. PHY7727084 and No. DMR81-17182.

\footnotetext{
${ }^{1}$ R. E. Peierls, Quantum Theory of Solids (Oxford, Univ. Press, London, 1955), p. 108.

${ }^{2}$ D. J. Scalapino and R. L. Sugar, Phys. Rev. B 24, 4295 (1981).

${ }^{3}$ T. Holstein, Ann. Phys. (N.Y.) 8, 325 (1959).

${ }^{4}$ W. P. Su, J. R. Schrieffer, and A. J. Heeger, Phys. Rev. B 22, 2099 (1980).

${ }^{5}$ J. E. Hirsch, D. J. Scalapino, R. L. Sugar, and R. Blankenbecler, Phys. Rev. Lett. 47, 1628 (1981).

${ }^{6}$ E. Fradkin and J. E. Hirsch, unpublished.

${ }^{7}$ G. Beni, P. Pincus, and J. Kanamori, Phys. Rev. B 10, 1896 (1974).

${ }^{8} \mathrm{~W} . \mathrm{P}$. Su, to be published.

${ }^{9}$ J. E. Hirsch and D. J. Scalapino, unpublished.

${ }^{10}$ See V. J. Emery, in Highly Conducting One-Dimensional Solids, edited by J. Devreese, R. Evrard, and V. van Doren (Plenum, New York, 1979), and references therein.
}

\title{
Quantized Hall Conductance in a Two-Dimensional Periodic Potential
}

\author{
D. J. Thouless, M. Kohmoto, ${ }^{(a)}$ M. P. Nightingale, and M. den Nijs \\ Department of Physics, University of Washington, Seattle, Washington 98195 \\ (Received 30 April 1982)
}

\begin{abstract}
The Hall conductance of a two-dimensional electron gas has been studied in a uniform magnetic field and a periodic substrate potential $U$. The Kubo formula is written in a form that makes apparent the quantization when the Fermi energy lies in a gap. Explicit expressions have been obtained for the Hall conductance for both large and small $U / \hbar \omega_{c}$.

PACS numbers: 72.15.Gd, 72.20. $\mathrm{Mg}, 73.90 .+\mathrm{b}$
\end{abstract}

The experimental discovery by von Klitzing, Dorda, and Pepper ${ }^{1}$ of the quantization of the Hall conductance of a two-dimensional electron gas in a strong magnetic field has led to a number of theoretical studies of the problem..$^{2-6}$ It has been concluded that a noninteracting electron gas has a Hall conductance which is a multiple of $e^{2} / h$ if the Fermi energy lies in a gap between Landau levels, or even if there are tails of localized states from the adjacent Landau levels at the Fermi energy. However, it can be concluded from
Laughlin's ${ }^{2}$ argument that the Hall conductance is quantized whenever the Fermi energy lies in an energy gap, even if the gap lies within a Landau level. For example, it is known that if the electrons are subject to a weak sinusoidal perturbation as well as to the uniform magnetic field, with $\varphi=p / q$ magnetic - flux quanta per unit cell of the perturbing potential, each Landau level is split into $p$ subbands of equal weight. ${ }^{7}$ One might expect each of these subbands to give a Hall conductance equal to $e^{2} / p h$, and that is what the clas- 
sical theory of the Hall current suggests, but according to Laughlin each subband must carry an integer multiple of the Hall current carried by the entire Landau level. This result appears even more paradoxical when it is realized that $p$, the number of subbands, can become arbitrarily large by an arbitrarily small change of the flux density. This paper contains a calculation of the Hall conductance for such a system, both in the limit of a weak periodic potential and in the tight-binding limit of a strong periodic potential. We have derived explicit expressions for the Hall currents carried by the various subbands, and show how the paradox is resolved.

We consider electrons in a potential $U(x, y)$ which is periodic in $x, y$ with periods $a, b$, and in a uniform magnetic induction $B$ perpendicular to the plane of the electrons. The band structure of such a system depends critically on $\varphi=a b e B / h$, which is the number of flux quanta per unit cell. We take $\varphi$ to be a rational number $p / q$; the behavior for irrational values of $\varphi$ can be deduced by taking an appropriate limit. We use the Landau gauge in which the vector potential has components $(0, e B x)$. In this gauge the eigenfunctions of the Schrödinger equation can be chosen to satisfy the generalized Bloch condition

$$
\psi_{k_{1} k_{2}}(x+q a, y) \exp \left(-2 \pi i p y / b-i k_{1} q a\right)=\psi_{k_{1} k_{2}}(x, y+b) \exp \left(-i k_{2} b\right)=\psi_{k_{1} k_{2}}(x, y),
$$

where $k_{1}$ (modulo $2 \pi / a q$ ) and $k_{2}$ (modulo $2 \pi / b$ ) are good quantum numbers. ${ }^{8}$ We can now define functions $u_{k_{1} k_{2}}=\psi_{k_{1} k_{2}} \exp \left(-i k_{1} x-i k_{2} y\right)$ which satisfy the generalized periodic boundary conditions

$$
u_{k_{1} k_{2}}(x+q a, y) e^{-2 \pi i p y / b}=u_{k_{1} k_{2}}(x, y+b)=u_{k_{1} k_{2}}(x, y) \text {, }
$$

and are eigenfunctions of a Hamiltonian

$$
\hat{H}\left(k_{1}, k_{2}\right)=\frac{1}{2 m}\left(-i \hbar \frac{\partial}{\partial x}+\hbar k_{1}\right)^{2}+\frac{1}{2 m}\left(-i \hbar \frac{\partial}{\partial y}+\hbar k_{2}-e B x\right)^{2}+U(x, y) .
$$

The components of the velocity operator are then given by $\hbar^{-1}$ times the partial derivatives of $\hat{H}$ with respect to $k_{1}, k_{2}$.

There are two quite different approaches to the problem of calculating the Hall conductance $\sigma_{\mathrm{H}}$. Laughlin ${ }^{2}$ and Halperin ${ }^{6}$ have studied the effects produced by changes in the vector potential on the states at the edges of a finite system. By this technique the quantization of the conductance is made explicit, but it is not obvious that the result is insensitive to boundary conditions. An alternative approach is to use the Kubo formula for a bulk two-dimensional conductor. In previous work using this method $^{3-5}$ it has not been made obvious that an integer value for the conductance must be obtained.

Because of the relation between the velocity operator and the derivatives of $\hat{H}$, the Kubo formula can be written as

$$
\sigma_{\mathrm{H}}=\frac{i e^{2}}{A \sigma^{\hbar}} \sum_{\epsilon_{\alpha}<E_{\mathrm{F}}} \sum_{\epsilon_{\beta}>E_{\mathrm{F}}} \frac{\left(\partial \hat{H} / \partial k_{1}\right)_{\alpha \beta}\left(\partial \hat{H} / \partial k_{2}\right)_{\beta \alpha}-\left(\partial \hat{H} / \partial k_{2}\right)_{\alpha \beta}\left(\partial \hat{H} / \partial k_{1}\right)_{\beta \alpha}}{\left(\epsilon_{\alpha}-\epsilon_{\beta}\right)^{2}},
$$

where $A_{0}$ is the area of the system and $\epsilon_{\alpha}, \epsilon_{\beta}$ are eigenvalues of the Hamiltonian. This can be related to the partial derivatives of the wave functions $u$, and gives

$$
\begin{aligned}
\sigma_{\mathrm{H}} & =\frac{i e^{2}}{2 \pi h} \sum \int d^{2} k \int d^{2} r\left(\frac{\partial u^{*}}{\partial k_{1}} \frac{\partial u}{\partial k_{2}}-\frac{\partial u^{*}}{\partial k_{2}} \frac{\partial u}{\partial k_{1}}\right) \\
& =\frac{i e^{2}}{4 \pi h} \sum \oint d k_{j} \int d^{2} r\left(u^{*} \frac{\partial u}{\partial k_{j}}-\frac{\partial u^{*}}{\partial k_{j}} u\right),
\end{aligned}
$$

where the sum is over the occupied electron subbands and the integrations are over the unit cells in $r$ and $k$ space. The integral over the $k$-space unit cell has been converted to an integral around the unit cell by Stokes's theorem. For nonoverlapping subbands $\psi$ is a single-valued analytic function everywhere in the unit cell, which can only change by an $r$-independent phase factor $\theta$ when $k_{1}$ is changed by $2 \pi / a q$ or $k_{2}$ by $2 \pi / b$. The integrand reduces to $\partial \theta / \partial k_{j}$. The integral is $2 i$ times the change in phase around the unit cell and must be an integer multiple of $4 \pi i$.

The problem of evaluating this quantum number remains. We have considered the potential

$$
U(x, y)=U_{1} \cos (2 \pi x / a)+U_{2} \cos (2 \pi y / b),
$$

both in the limit of a weak periodic potential $(|U|$ $\left.\ll \hbar \omega_{c}\right)$ and in the tight-binding limit of a strong periodic potential. In the weak-potential limit the wave function can be written as a superposition of the nearly degenerate Landau functions in 
the same Landau level $N$ :

$$
u_{k_{1} k_{2}}=\sum_{n=1}^{p} d_{n} \sum_{l=-\infty}^{\infty} \chi_{N}\left(x-\frac{\hbar k_{2}}{e B}-l q a-\frac{n q a}{p}\right) \exp \left[-i k_{1}\left(x-l q a-\frac{n q a}{p}\right)+2 \pi i y \frac{(l p+n)}{b}\right],
$$

where $\chi_{N}$ is the appropriate oscillator wave function. Since the term $U_{1} \cos (2 \pi x / a)$ is diagonal in the Landau functions and $U_{2} \cos (2 \pi y / b)$ changes the value of $n$ by unity, the amplitudes $d_{n}$ satisfy the secular equation of the form

$$
V \exp \left(-i q a k_{1} / p\right) d_{n-1}+2 V^{\prime} \cos \left(q b k_{2} / p+2 \pi n q / p\right) d_{n}+V \exp \left(i q a k_{1} / p\right) d_{n+1}=E d_{n},
$$

with $V$ and $V^{\prime}$ proportional to $U_{2}$ and $U_{1},{ }^{7}$ and with $d_{n+p}=d_{n}$. This is known as Harper's equation. ${ }^{9}$ Its spectrum has been studied in detail by Hof stad$\operatorname{ter}^{10}$ for the iostropic case $V=V^{\prime}$. We have made considerable use of his results.

Numerical solution of the eigenvalue problem (8) and direct substitution in Eqs. (7) and (5) are possible but laborious. We have done this for a number of cases. The results obtained in this way are entirely concordant with the general results we have obtained by examining the limit $V$ $\ll V^{\prime}$. The quantum number of a subband cannot change unless the gaps close up; we believe that none of the gaps closes when $V / V^{\prime}$ is varied, but we have no proof of this. For small $V / V^{\prime}$ only one component $d_{n}$ is appreciably different from zero, except for $q b k_{2}$ close to a multiple of $\pi$, where there is a changeover in the dominant component. These values of $k_{2}$ are the locations of the energy gaps which have opened for small $V /$ $V^{\prime}$. In the interval $0 \leqslant k_{2}<2 \pi / b$, the $r$ th gap repeats itself $q$ times, at values of $q b k_{2}$ equal to an odd (even) multiple of $\pi$, if $p-r$ is odd (even). Here the $r$ th and $(r+1)$ th band change their dominant component $d_{n}$ according to $n_{r} \rightarrow n_{r}+s_{r}, n_{r+1}$ $\rightarrow n_{r+1}-s_{r}$. The value of $s_{r}$ is independent of $k_{2}$ and determined by the Diophantine equation

$$
r=s_{r} q+t_{r} p
$$

where $|s| \leqslant p / 2$. The $r$ th gap is of order $(V /$ $\left.V^{\prime}\right)^{\left|s_{r}\right|}$ : Each time, the wave function of band $r$ picks up a phase from the off-diagonal terms in Eq. (8): $q$ times $q a k_{1} s_{r} / p$ and $q$ times $-q a k_{1} s_{r-1} /$ $p$. The total phase change in $d_{n}$ is

$$
\begin{aligned}
& \begin{aligned}
d_{n}\left(k_{1}, k_{2}\right) & =d_{n}\left(k_{1}+2 \pi / a q, k_{2}\right) \\
& =d_{n}\left(k_{1}, k_{2}+2 \pi / b\right) \exp \left[-i \theta_{d}\left(k_{1}\right)\right]
\end{aligned} \\
& \theta_{d}\left(k_{1}\right)=q a k_{1}\left(t_{r-1}-t_{r}\right)+q a k_{1} / p .
\end{aligned}
$$

The term $q a k_{1} / p$ in $\theta_{d}$ cancels against the phase change which is already explicit in Eq. (7). It represents the classical Hall current. The total Hall current carried by the $r$ th band is quantized according to $\sigma_{\mathrm{H}}=\left(e^{2} / h\right)\left(t_{r}-t_{r-1}\right)$. If the Fermi surface is located in the $r$ th gap of the $N$ th Landau level, the total Hall conductance is equal to

$$
\sigma_{\mathrm{H}}=\left(e^{2} / h\right)\left(t_{r}+N-1\right),
$$

with $t_{r}$ the solution of Eq. (9). This has an unambiguous solution, expect for the case of $p$ even, $r=\frac{1}{2} p$, where there is no gap.

For $q=1$ this gives $t=0$ in the first half of the Landau level and $t=1$ in the second half, so that only the central subband of each Landau level carries the Hall current. For $q=2$, the values of $t$ are alternately 1 and 0 , so that alternate subbands carry \pm 1 times the Hall current of the Landau level. In general each subband carries one of two possible Hall currents which differ from one another by $q$ units. For example, in the case $q / p$ $=\frac{11}{7}$, the first 11 values of $t$ are $-3,5,2,-1$, $-4,4,1,-2,6,3,0$, so that the Hall current is proportional to -3 or 8 in each subband.

Figure 1 gives an intuitive explanation of the results. The abscissa represents the variable $k_{2}$, or equivalently the position of the center coordinate of the state in the $x$ direction, while the ordinate gives the energy as a function of this variable. Application of an electric field in the $y$ direc-

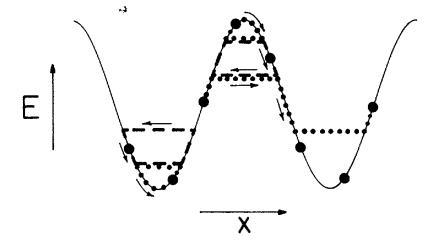

(a)

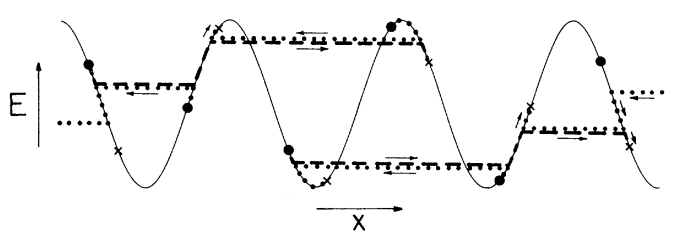

(b)

FIG. 1. Motion of electrons in the $x$ direction under the influence of an electric field in the $y$ direction for $V \ll V^{\prime}$, for the two cases (a) $\varphi=5$ and (b) $\varphi=\frac{5}{3}$. 


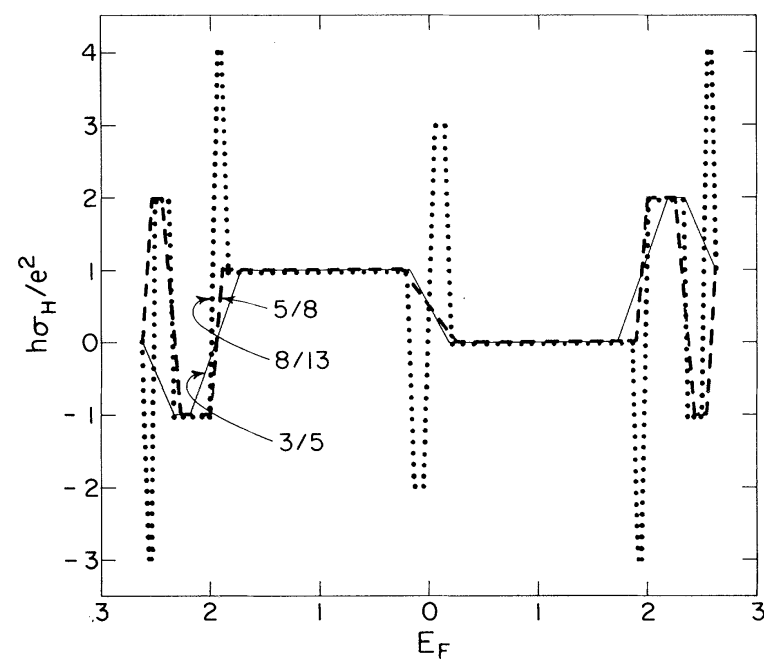

FIG. 2. Hall current as a function of Fermi energy for a weak isotropic substrate. Truncating the continued-fraction expansion of $\frac{1}{2} \sqrt{5}-\frac{1}{2}$, we obtained $q / p=\frac{3}{5}$, $\frac{5}{8}, \frac{8}{13}, \ldots$. Within the energy bands, the Hall current was not calculated; the interpolation merely serves as a guide to the eye.

tion gives a steady change of $k_{2}$, and therefore a steady motion of a representative point along the $E\left(k_{2}\right)$ curve, until the energy gets very close to the energy of a state whose $k_{2}$ value differs by a multiple of $2 \pi / b$. Then the two states exchange places rather than crossing in energy. Figure 1 (a) shows the case $p=5, q=1$. Four of the orbits are closed and give no motion in the $x$ direction, while a Hall current of +1 is given by the middle orbit. Figure 1 (b) shows $p=5, q=3$, where three orbits give a negative Hall current equal to -1 , and the other two give a positive current +2 .

In the opposite limit of a strong potential $U$ the same Eq. (8) can be obtained, where $V$ and $V^{\prime}$ are the tight-binding matrix elements that take an electron from a site to its neighbors in the $x$ and $y$ directions, and $\varphi$ is replaced by $1 / \varphi$, so that $p$ and $q$ are interchanged. ${ }^{9,10}$ The result is that the Hall conductance $\sigma_{\mathrm{H}}$ is equal to $t e^{2} / h$. Again, $t$ is given by Eq. (9), but now $s$ is unconstrained and $t$ must lie between $-\frac{1}{2} q$ and $+\frac{1}{2} q$. In the case $p / q=\frac{7}{11}$, the values of $t$ are $-3,5,2,-1,-4$, $4,1,-2,-5,3,0$, which are the same as the weak-potential limit gives except for the ninth gap, where +6 has been replaced by -5 . This pattern is easy to understand, as $p$ subbands constitute one Landau level, so we get a Hall current $t$ if we count up $t p$ (modulo $q$ ) levels from the bottom of the band, and $-t$ if we count down $t p$ (modulo $q$ ) levels from the top of the band.

It is generally true that the larger the integer part of $t p / q$, the smaller will be the corresponding energy gap. The complexity of the structure is associated with the smaller energy gaps as illustrated in Fig. 2; for these smaller energy gaps, the electric field has to be very small for linear response theory to be valid. Stronger fields will cause tunneling across the gap. It is this restriction, and corresponding restrictions on disorder and deviations from the simple sinusoidal potential, that resolves the paradox of the sensitivity of the Hall conductance to the precise value of $\varphi$. In fact, as Hof stadter ${ }^{10}$ and Wannier ${ }^{11}$ have shown, each energy gap persists over a continuous range of $\varphi$. The Hall conductance is constant in a particular energy gap, even for irrational values of $\varphi$.

Streda ${ }^{12}$ has recently obtained a result for the Hall conductance that is in agreement with our results.

This work was supported by the National Science Foundation under Grant No. DMR7920785.

(a) Present address: Department of Physics, University of Illinois, Urbana, Ill. 61801.

${ }^{1} \mathrm{~K}$. von Klitzing, G. Dorda, and M. Pepper, Phys. Rev. Lett. 45, 494 (1980).

${ }^{2}$ R. B. Laughlin, Phys. Rev. B 23, 5632 (1981).

${ }^{3}$ H. Aoki and T. Ando, Solid State Commun. $\underline{38}, 1079$ (1981).

${ }^{4}$ R. E. Prange, Phys. Rev. B $\underline{23}, 4802$ (1981).

${ }^{5}$ D. J. Thouless, J. Phys. C 14, 3475 (1981).

${ }^{6} \mathrm{~B}$. I. Halperin, to be published.

${ }^{7}$ A. Rauh, G. H. Wannier, and G. Obermair, Phys. Status Solidi (b) 63,215 (1974).

${ }^{8} \mathrm{~J}$. Zak, Phys. Rev. 134, A1607 (1964).

${ }^{9}$ P. G. Harper, Proc. Phys. Soc., London, Sect. A 68 , 874 (1955).

${ }^{10}$ D. Hofstadter, Phys. Rev. B 14, 2239 (1976).

${ }^{11}$ G. H. Wannier, Phys. Status Solidi (b) $\underline{88}, 757$ (1978).

${ }^{12} \mathrm{P}$. Streda, to be published. 